\title{
Quantitative Single-Cell Gene Expression Measurements in Bacteria Using Time-Lapse Microscopy
}

\author{
Mary J. Dunlop ${ }^{1}$ \\ ${ }^{1}$ School of Engineering, University of Vermont, Burlington, VT, USA.
}

Recent research has uncovered many examples of genetically identical organisms that display distinct, dynamic phenotypes. For example, single-cell studies have revealed cell-to-cell variability in expression of key regulatory proteins in bacteria [1,2] and stress response pathways in yeast [3-5]. Although methods such as flow cytometry can measure population diversity at the single-cell level, in order to understand the origins of cell-to-cell variability in gene expression, it is necessary to track single cell lineages through time. Time-lapse microscopy, using fluorescent reporters and image processing is an ideal way to achieve this detailed, dynamic history of gene expression for many cells within a microcolony [6,7]. An example is shown in Fig. 1. Here, we summarize methods for obtaining time-lapse images of growing cells and will describe the image analysis tools we have developed.

For bacteria such as Escherichia coli, we use an automated fluorescence microscope (Nikon Ti-E with Nikon Elements software) with a 100x objective in a temperature controlled environment. Images are obtained in phase contrast and fluorescence channels at regular intervals, such as every 10 minutes over the course of 12-24 hours. In advance, cultures are grown to early exponential phase $\left(\mathrm{OD}_{600}=0.1\right)$ and then diluted back 1:500 into fresh medium with inducers and antibiotics, as appropriate. A 1 $\mu$ l drop of this diluted culture is pipetted onto an agarose pad and allowed to dry before placement on a glass surface for imaging (e.g. WillCo glass bottom dish). Agarose pads are prepared using 1.5\% low melting point agarose and an optically clear growth medium. Pads are made by pipetting the agarose mixture onto a cover slip and placing another cover slip on top. After the agarose has solidified, the top coverslip is slid back and small $(5 \mathrm{~mm} \times 5 \mathrm{~mm})$ pads are cut and used as the surface on which to grow cells. This setup constrains growth of the bacterial microcolony to two dimensions, which enables subsequent image processing.

The phase contrast image is used for post-processing to identify cells and the fluorescence values are extracted based on the locations of cells in the phase contrast image. We developed custom Matlab software which has three main parts: (1) Identification of the cells in the phase contrast image. This can be achieved using background subtraction and segmentation algorithms that are specific to the type of cells being imaged. (2) Tracking of cells from frame to frame. The software tracks cell lineages, starting with a parent, through all subsequent frames until cell division. This step is automated by analyzing the centroid of each cell from frame to frame and looking for cell division events by tracking the major axis length and cell area across frames. (3) Data extraction. The cell locations in the phase contrast image are used to extract fluorescence data for each cell. These data are then compiled for the entire microcolony.

Examples with applications in synthetic and natural gene circuits will be discussed.

\section{References}

1. Choi PJ, Cai L, Frieda K, Xie S (2008) A stochastic single-molecule event triggers phenotype switching of a bacterial cell. Science (New York, NY) 322: 442-446. 
2. Locke JCW, Young JW, Fontes M, Hernández Jiménez MJ, Elowitz MB (2011) Stochastic pulse regulation in bacterial stress response. Science (New York, NY) 334: 366-369.

3. Cai L, Dalal CK, Elowitz MB (2008) Frequency-modulated nuclear localization bursts coordinate gene regulation. Nature 455: 485-U416.

4. Hansen AS, O\&apos; Shea EK (2013) Promoter decoding of transcription factor dynamics involves a trade-off between noise and control of gene expression. Molecular Systems Biology 9: 704.

5. Hao N, Budnik BA, Gunawardena J, O'Shea EK (2013) Tunable signal processing through modular control of transcription factor translocation. Science (New York, NY) 339: 460-464.

6. Locke JCW, Elowitz MB (2009) Using movies to analyse gene circuit dynamics in single cells. Nature Reviews Microbiology 7: 383-392.

7. Young JW, Locke JCW, Altinok A, Rosenfeld N, Bacarian T, et al. (2012) Measuring single-cell gene expression dynamics in bacteria using fluorescence time-lapse microscopy. Nature Protocols 7: $80-88$.

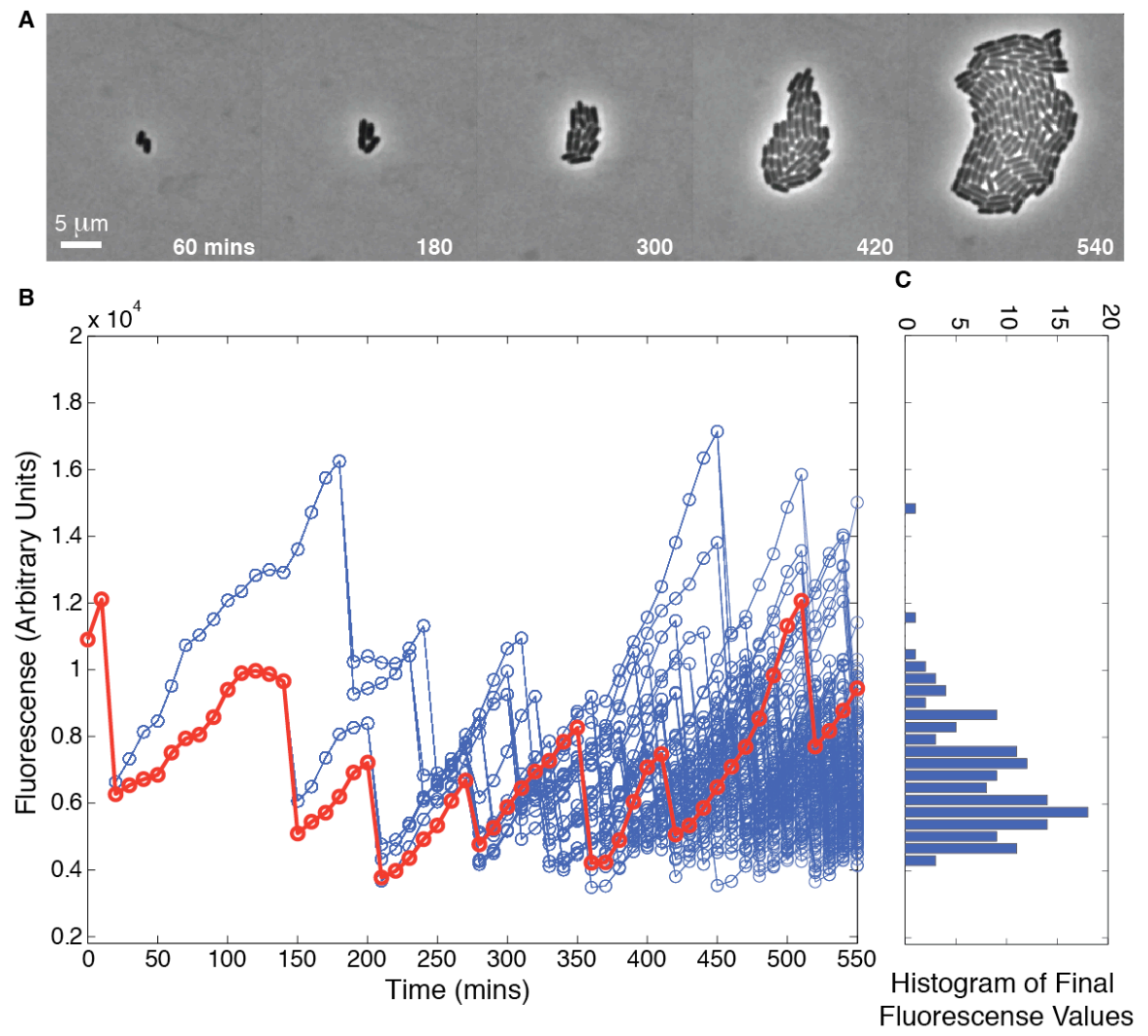

Figure 1. Cell-cell variability in E. coli gene expression. (a) Snapshots of single cells. The microcolony originated from a single cell. Time and length scales are indicated on the figure. (b) Fluorescent protein expression levels versus time. Blue lines are data for all cells. The red trace follows a single lineage. Sharp drops in expression are cell division events. (c) Distribution of final flourescent protein expression levels shows variability in gene expression. 